\title{
Automatic segmentation of spinal cord diffusion MR Images for disease location finding
}

\author{
Sk. Hasane Ahammad, V. Rajesh, A. Neetha, Sai Jeesmitha.B, A. Srikanth \\ Department of Electronics and Communication Engineering, Koneru Lakshmaiah Education Foundation, India
}

\begin{tabular}{l}
\hline Article Info \\
\hline Article history: \\
Received Dec 21, 2018 \\
Revised Mar 2, 2019 \\
Accepted Apr 25, 2019
\end{tabular}

\section{Keywords:}

MRI images

Segmentation

Spine

Weighted-diffusion

\begin{abstract}
Dissemination weighted MR imaging may build the affectability and explicitness of MR imaging for certain pathologic states of the spinal rope yet is once in a while performed as a result of a few specialized issues. We consequently tried a novel stage explored turn reverberation dispersion weighted interleaved reverberation planar imaging arrangement in seven sound volunteers and six patients with intramedullary injuries. We performed dispersion weighted MR imaging of the spinal string with high spatial goals. Distinctive examples of dissemination irregularities saw in patient investigations bolster the conceivable symptomatic effect of dispersion weighted MR imaging for ailments of the spinal string. MR imaging has turned into the system of decision for imaging the spinal rope on account of a high affectability for pathologic intra medullary changes. In any case, the explicitness of anomalies oftentimes lingers behind when utilizing just regular MR arrangements. Dissemination weighted MR imaging guarantees to supply additional data in light of trademark changes of the clear dispersion coefficient, for example, those showed in intense ischemia, tumors, or sores related among numerous sclerosis. To date, the indicative commitment of dispersion weighted MR imaging has been concerted essentially in the cerebrum since dissemination weighted MR imaging of the spine is in detail every one the more requesting. Both the little size of the spinal rope and movement-initiated antiquities must be considered. We in this manner built up another examination strategy and tried its unwavering quality and potential for adding to the symptomatic workup of patients with spinal rope indications.
\end{abstract}

Copyright $\odot 2019$ Institute of Advanced Engineering and Science. All rights reserved.

\section{Corresponding Author:}

Sk Hasane Ahammad,

Department of Electronics and Communication Engineering,

Koneru Lakshmaiah Education Fondation,

Guntur, India-522502.

Email: ahammad@kluniversity.in

\section{INTRODUCTION}

For dissemination imaging, we utilized a stage explored interleaved reverberation planar imaging technique [1]. The dispersion weighted successions were RR-gated by utilizing the plethysmographic motion from a finger-beat oximeter activated from consistently R-top to maintain a strategic distance from relics from the development of the spinal string and the throb of CSF [2]. In addition, to avoid impacts of moving structures, sideways immersion pieces were put in the pharynx and thoracic cavern locale. Furthermore, a ghastly fat-immersion beat was utilized to stifle the curios emerging from lipid tissue (ie, synthetic move). In view of divided k-freedom obtaining, the reverberation time- move method was connected to decrease balances of the stage mistake employment in k-space that generally guide to ghosting ancient rarities [3-4]. The succession parameters for dissemination weighted MR imaging were as per the subsequent: $~ 1500 / 87 / 4$ (TR/TE/excitations); imaging network, $192 \times 256$; pasture of view, $161 \times 230 \mathrm{~mm}$; limited Fourier imaging; 
amount of angle echoes per reverberation planar imaging interleaf factor, 11; area thickness/hole, 3.5/0.35 $\mathrm{mm}$; and figure of sections. The term, $\delta$, of one dispersion angle beat was $28 \mathrm{~ms}$. The time between the main edges of both dissemination angle beats, $\Delta$, was $37 \mathrm{~ms}$. B0-inhomogeneities were redress by schemes for a seller explicit programmed shimming strategy [5-6]. For stage route, the nonlinear strategy introduced in [7] was utilized, which gives phase amendment to both translational and rotational development. The revision was performed following information securing amid picture recreation. The technique that was utilized performs stage revision in mixture space after 1D-Fourier change of the gained information in readout heading. One guide projection in mixture space was picked as a kind of perspective view for computing the relative stage bothers of the rest of the perspectives, mirroring the irregular movements of the member [8-9].

\section{LITERATURE SURVEY}

Various sclerosis (MS) is an interminable insusceptible interceded illness of the focal sensory scheme, through changeable scientific articulation [10]. The pathologic sign of M/S is the event of central regions of incendiary demyelization inside the mind along with spinal string, recognized as sores. M-S injuries display changeable quantity of demyelisation, axonal damage also misfortune, remyelination, plus gliosis [11]. Weakened axonal transmission frequently reasons engine, tangible, optical, in addition to subjective injury. Clinicians moreover analysts widely utilize traditional M-R-I (e.g., T2-prejudiced) to nonobtrusively measure the injury load in reality [12-14]. The investigation of spinal rope sores have as of belatedly accumulated premium agreed its possible incentive on behalf of conclusion moreover anticipation of MS. Besides, spinal line decay is basic in MS, in addition to the measurement of such decay is clinically applicable also relates among experimental inability [15-18]. Thusly, separation of the spinal string along with M-S sores restricted inside it (intramedullary sores) is a typical strategy to quantitatively review the basic uprightness of this part of the focal sensory scheme in M.S patients. Be that as it may, manual division is tedious and experiences intra-and between rater inconstancy. Consequently, there is a requirement for powerful and programmed division devices in support of the spinal line along with the intramedullary $M * S$ injuries [19-20].

Different programmed spinal rope division techniques contain be planned in the previous couple of lifetime, counting dynamic forms as well as surface-supported methodologies. While these techniques have demonstrated great execution, they regularly necessitate a particular area of intrigue also additionally are restricted in the direction of a particular complexity moreover goals [21-23]. Also, the absence of approval alongside multi-site information before belongings among spinal line harm has restricted their submission in vast experimental multi-location thinks about. Automatic spinal string division is hard to accomplish heartily also precisely over the expansive scope of spinal rope outline, lengths, also pathologies; and crosswise over factor picture measurements, goals, introductions, differentiations, as well as ancient rarities (for instance weakness, movement, synthetic move, ghosting, obscuring, Gibbs) [24-26]. Figure 1 shows these difficulties, portraying the heterogeneity every now and again saw in multi-site clinical spinal string informational collections.

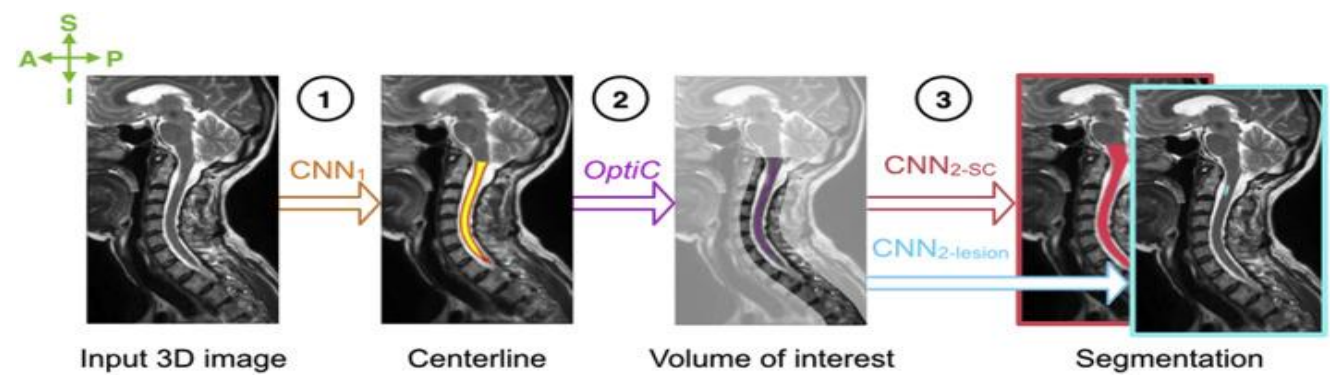

Figure 1. Stream of work

\section{RELATED WORK}

The spinal line is regularly influenced by decay and additionally injuries in various sclerosis-(M*S) patients. Division of the spinal string also sores commencing M/R/I information gives extent of harm, which be solution criterion in favour of the finding, guess, as well as longitudinal observing in M/S. Computerizing this mission wipes out between rater changeability and expands the productivity of substantial throughput investigation pipelines. Strapping and dependable division crosswise over multi-site spinal string information 
is testing a result of the huge changeability identified with procurement parameters and picture antiques. Purposely, an accurate representation of sore is impede beside an generous heterogeneity of damage discriminate, dimension, region, moreover figure. The objective of this examination was to build up a completely programmed structure-vigorous to fluctuation in mutually picture limitation along with quantifiable circumstance-in support of division of the spinal line in addition to intramedullary M,S sores starting regular M/R/I information of $\mathrm{M} * \mathrm{~S}$ moreover non-M/S belongings. Production of 1042 matter (459 sound gearstick, $471 \mathrm{M} / \mathrm{S}$ patients, as well as 112 among previous spinal pathologies) be incorporated into this multi-site think about $(\mathrm{n}=30)$. Information traversed 3 differentiations $(\mathrm{T} 1-, \mathrm{T} 2-$, and T2-slanted) in favour of a sum of $1943 \mathrm{vol}$ along with included substantial heterogeneity as far as goals, beginning, inclusion, as well as experimental circumstances. The anticipated line also sore programmed division move towards depends on a grouping of 2 Convolutional Neural Networks (CNNs) [8] . To supervise the small amount of spinal procession or else potentially injury voxels contrast among anything is absent of the quantity, a initial C/N/N with $2 \mathrm{D}$ distended convolutions identifies the spinal filament centreline, trailed by a flash CNN amid 3D convolutions to facilitate portions the spinal string or potentially sores. CNNs were prepared freely with the Dice misfortune. At the point when thought about alongside physical division, our CNN- support methodology demonstrated a middle Dice of $95 \%$ versus $88 \%$ in favour of Prop Seg $(\mathrm{p} \leq 0.05)$, a best in class spinal line division strategy. As to separation on MS information, our structure gave a Dice of $60 \%$, a comparative amount contrast of $-15 \%$, also an injury insightful location affectability and accuracy of $83 \%$ and $77 \%$, separately. In this investigation, we acquaint a strong strategy with segment the spinal string in addition to intramedullary MS injuries on an assortment of M/R/I dissimilarity. The planned structure is open-foundation and promptly accessible in the Spinal string Toolbox.

The dispersion lessening (b-values) values utilized were roughly 0 and $709 \mathrm{~s} / \mathrm{mm}^{2}$. Dispersion weighted MR imaging judgment were acquired with dissemination weighting along every chief pivot (anteroposterior, left-right, and cephalocaudal) and with sagittal segment request. Further post preparing was performed disconnected utilizing an in-house committed MR picture handling programming. The evident dissemination coefficient, $\mathrm{ADC}$, was determined in support of every pixel premise as indicated by the accompanying trademark condition:

$$
\mathrm{ADC}=\frac{1}{\mathrm{~b}}\left(\ln \mathrm{S}_{0}-\ln \mathrm{S}_{\mathrm{b}}\right),
$$

Where $\mathrm{b}$ is the dissemination weighting feature, $\mathrm{Sb}$ is the dispersion weighted standard, and $\mathrm{S} 0$ is the flag force when the dispersion angles were killed. Also, in our volunteer investigation, the hint of the dispersion tensor was utilized, which assesses the accompanying:

$$
\operatorname{Tr}(\mathbf{D})=\sum_{\mathrm{i} \in \mathrm{AP}, \mathrm{LR}, \mathrm{CC}} \mathrm{ADC}_{\mathrm{i}}
$$

Where AP, LR, and CC, demonstrate dissemination weighting along the anteroposterior, left-right, and cephalocaudal headings. Eventually, follow weighted dissemination pictures, STrace, were determined by the accompanying condition:

$$
\mathrm{S}_{\text {Trace }}=\sqrt[3]{\mathrm{S}_{\mathrm{AP}} \mathrm{S}_{\mathrm{LR}} \mathrm{S}_{\mathrm{CC}}}
$$

\section{PROPOSED WORK}

Programmed division structure. (1) recognition of the spinal line by C-N-N1 which yields a warmth map (red/to/yellow) of the spinal string area, (2) computation of the spinal rope focus line (pink) commencing the spinal rope warmth map, as well as taking out of 3D fixes in a quantity of enthusiasm encompassing the spinal rope focus line, (3) separation of the spinal string (red) through C/N/N/2-S/C, as well as of sores (azure) beside CNN. SC: Spinal rope; C*N/N: Convolutional Neural Network; S:-Superior; I:-Inferior; An:-Anterior; P:-Posterior appeared in Figure 1 and 2.

CNNs can without much of a stretch overfit due to two principle highlights of our informational index: (I) the elevated division unevenness because of the modest figure of voxels marked as constructive ( $\sim 0.34 \%$ in favour of spinal string, $\sim 0.01 \%$ in favour of sores), as well as (ii) the set amount of available name pictures. To avoid over fitting, the projected system tear the education plan hooked on 2 phases, every surround a $\mathrm{C} / \mathrm{N} / \mathrm{N}$. The main period comprises of distinguishing the focal point of the spinal line $(\mathrm{C} / \mathrm{N} / \mathrm{N} / 1)$ furthermore harvest the picture approximately it, whereas the instant point sections the spinal rope $(\mathrm{C}-\mathrm{N} * \mathrm{~N}-$

Automatic segmentation of spinal cord diffusion MR Images for disease location... (Sk. Hasane Ahammad) 
2-S-C) as well as additionally the $\mathrm{M} * \mathrm{~S}$ injury (CNN2-sore). Note with the aim of $\mathrm{CNN}^{*} 2-\mathrm{S}-\mathrm{C}$ in addition to $\mathrm{C} / \mathrm{N} / \mathrm{N}^{*} 2$-injury be autonomously prepared also canister subsist run independently. The inspiration driving the consecutive methodology is that CNN's include be appeared en route for become familiar with a progressive portrayal of the gave information because the mountain deposit of convolution channels be custom fitted on the way to the ideal division. The planned successive structure guarantees with the purpose of (I) CNN/1 be trained channels to separate connecting the hub fixes that enclose spinal rope voxels [10-15] versus pieces with the intention of don't, (ii) whilst CNN-2-SC (moreover CNN/*2-injury) is prepared to upgrade a lot of channels customized to the spinal rope (in addition to the injuries) since preparing squares based on the spinal rope.

Programmed pre handling steps incorporate taking after to $0.5 \mathrm{~mm}$ isotropic pictures (in view of fundamental improvements), and network re-introduction (RPI, for instance Appropriate to-absent, Posteriorto-foremost, Inferior-to-unrivaled).

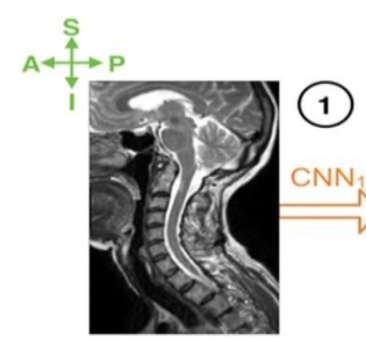

Input 3D image

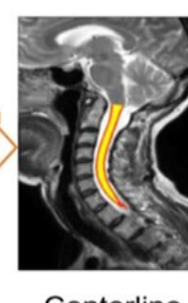

Centerline

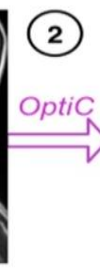

Volume of interest
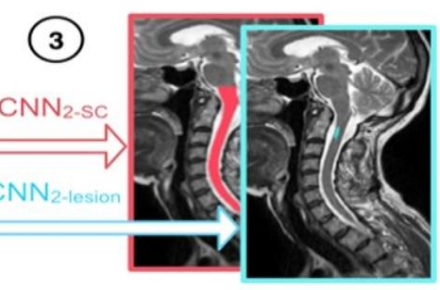

Segmentation

Figure 2. Mirror CNN image

\subsection{Spinal Cord Centreline Detection}

Identification of the string centreline (1 Figure 3, stage 1 ) is accomplished among a $2 / \mathrm{D} \mathrm{C}^{*} \mathrm{~N} * \mathrm{~N}$ (C/N/N/1), during every one cross-sectional cut of the information amount. On behalf of every info amount, we separate $2 \mathrm{D}$ squares $(96 \times 96)$ beginning the cross-sectional cuts. We figured the indicate power in addition to benchmark departure over the preparation patches, to standardize each and every one the handled scrap (for instance zero indicate as well as element fluctuation), counting the approval along with difficult patches.

$\mathrm{C}-\mathrm{N} / \mathrm{N} 1$ plan be familiar commencing the U-net manufacturing by lessening the behind example layers starting 4 to 2 layers, also through supplanting standard convolutions among widened convolutions in the contracting method. Quickly, widened complexity is a difficulty among characterized holes, which gives an exponential extension of the responsive view with a direct increment of parameters. The inspiration driving the utilization of expanded convolutions is to catch increasingly logical data (for example more extensive perspective of the contribution), with less parameters contrasted with a traditional arrangement, which includes extra downsampling layers. Primer examinations drove us to utilize a widening rate of three (for example a hole of two pixels for every contribution, as additionally showed in Figure 1 To decrease overfitting, Batch Normalization, amended straight initiation capacity, and Dropout (preparing with $p=0.2$ ) pursue every convolution layer.

Preparing of CNN1 be carry out on all difference informational index independently (for example three prepared models: T1-w, T2-w, also T2*-w), utilizing the Adam enhancer, among a erudition time of 0.0001 , a group dimension of 32, furthermore 100 ages. We utilized Dice misfortune for the misfortune work because of its cold-heartedness to high class irregularity, as favored by ongoing investigations managing this issue. We played out a broad information growth of the preparation tests, including moving $( \pm 10$ voxels toward every path), flipping, turn $\left( \pm 20^{\circ}\right.$ toward every path), and versatile distortions (twisting coefficient of 100, standard deviation of 16). Flexible changes were appeared to be proficient at expanding learning invariance and sensible variety in tissue Spinal string centerline extraction is accomplished by recreating a volume from the fix derivation of $\mathrm{CNN} 1$, where esteems demonstrate the level of certainty in regards to the spinal string area. Since CNN1 yields an expectation cover with sudden limits, we figure the Euclidean separation map from the CNN1 yield to help among spinal string centerline identification (red-to-yellow qualities in 2 Figure 3, stage 1). We gather the centerline commencing this spinal line remove map utilizing OptiC, a recently distributed quick worldwide bend streamlining calculation, which regularizes the centerline congruity the length of the Superior-to-Inferior pivot (pink centerline in 2 Figure 2, stage 2). 


\subsection{Spinal cord and MS lesions segmentation}

Dissection of the spinal line as well as the intra modularly sores are talented beside CNN-2-SC as well as $\mathrm{CNN}-2$, which are together 3/D CNN's investigative in a amount of eagerness surrounding the construed rope centreline. From every amount, we separate 3D fixes beside the spinal rope centreline (Figure 2, stage 2) among the accompanying sizes: $64 \times 64 \times 48$ for the spinal rope (for instance CNN2-SC) and $48 \times 48 \times 48$ for MS sores (for example CNN2-Lesion). In fundamental trials, we researched diverse fix sizes $(32 \times 32 \times 32,48 \times 48 \times 48,64 \times 64 \times 48$, also $96 \times 96 \times 48)$ furthermore chose a trade off stuck between the group lopsidedness, the danger of over fitting, also the computational expense. We concern a force standardization calculation on the stack square of every volume to homogenize the power circulations on an institutionalized power extend Finally, following indistinguishable procedure from in area 2, we standardize the fix powers by focusing the indicate plus normalizing the criterion departure. CNN2-SC as well as CNN2Lesion designs illustrate starting the 3D U-net plan nonetheless, we diminished the profundity of the U-shape from three to two, along these lines constraining the quantity of limitation along with the measure of recollection necessary on behalf of preparing. Preparing of CNN2-SC also CNN2-injury be likewise embraced in support of every one differentiation, despite the fact that CNN2-sore was prepared with MS information as it were. We prepared the mould utilizing the Adam enhancer, the Dice misfortune, the Dropout $(\mathrm{p}=0.4)$, and the accompanying parameters: a clump size of 4 , education velocity of $5 \times 10-5$, and all out quantity of ages of 300. Other than flipping activities, the information growth methodology incorporated little nearby disintegrations and enlargements of the manual sore limits, which serve to experiment the certainty of the system on abstract sore outskirts. Amid the induction organize, CNN2-SC[1619] as well as CNN2-Lesion freely fragment 3D patches separated commencing a difficult information. We relate a limit of 0.5 to the $\mathrm{C}-\mathrm{N}^{*} \mathrm{Ns}$ expectations previous to remaking a 3-D capacity (Figure 2, stage 4). The displayed system do not include extra post-handling.

\subsection{Implementation}

We executed the projected strategy in the Python 2.7 speech, utilizing Keras1(v2.6.0) along with TensorFlow2 (v1.3.0) libraries. The cipher of the CNNs executions is accessible on GitHub3 . Additionally, the introduced techniques are promptly accessible through the capacities sct_deepseg_sc and sct_deepseg_lesion as a feature of the Spinal Cord Toolbox (SCT) form v3.2.2 also elevated. These capacities are vigorous to several picture goals and introduction, just as number of cuts, notwithstanding for single pivotal cut pictures. CNN preparing was done on a solitary NVIDIA Tesla P100 GPU with 16 GB RAM memory and took around 6, 70, and $102 \mathrm{~h}$, for CNN1, CNN2-SC, furthermore CNN2-sore, individually. Preparing was halted when the preparation misfortune continued diminishing while the approval misfortune relentlessly expanded or settled down. In spite of the preparation which require towering computational authority, for example, that accessible by means of a $\mathrm{G} / \mathrm{P} / \mathrm{U}$, deduction (for exemplar division) can keep running in just a couple of minutes on a standard CPU.

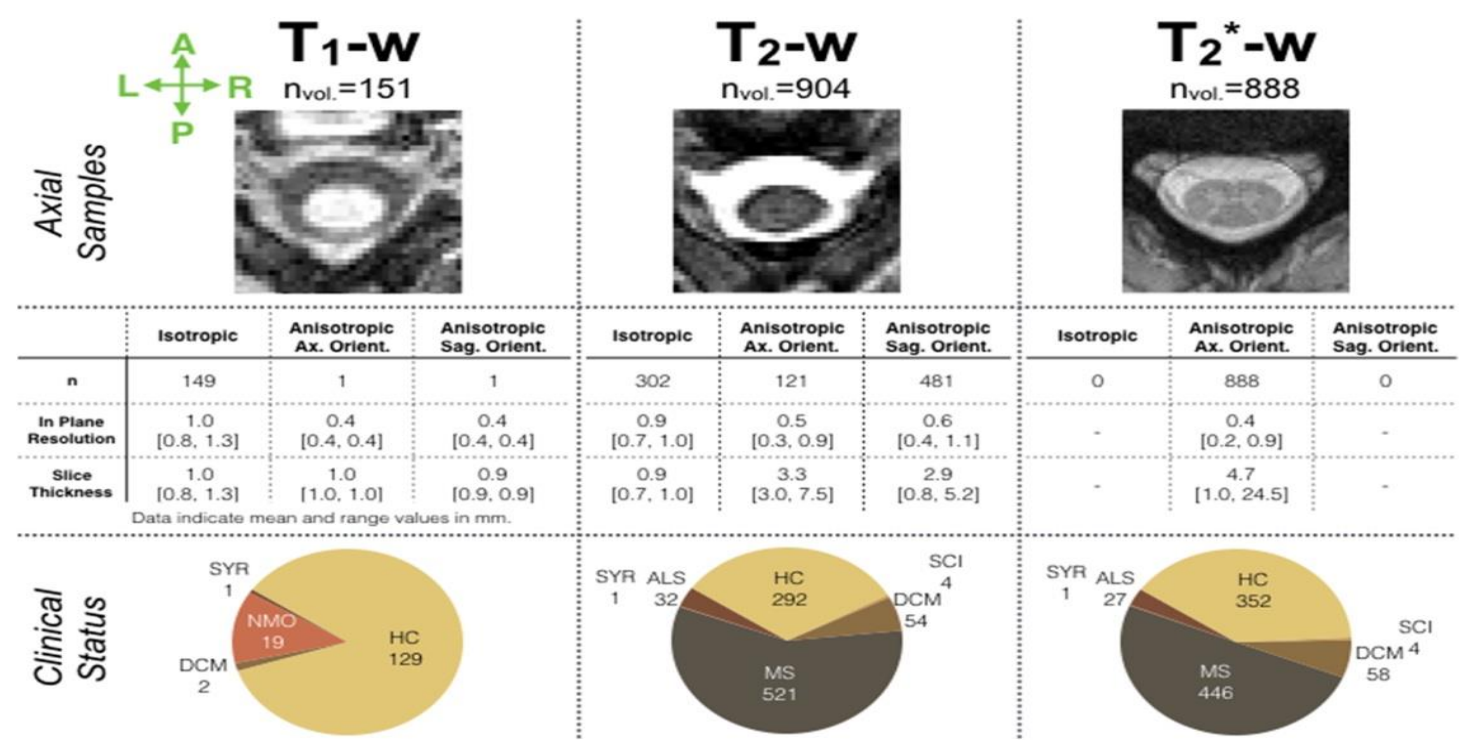

Figure 3. Disease location finding 


\subsection{Evaluation}

In support of every one complexity (for example T1-, T2-, T2*-weighted), the systems be prepared on $80 \%$ of the subjects, with $10 \%$ waited on behalf of approval and $10 \%$ for difficult (for example for results exhibited in segment 3.). Specifically, the testing informational collection contained information from two locales $(n=57)$, which be absent amid the preparation strategy, so as to assess the speculation of the pipeline to new picture highlights.

\subsubsection{Spinal cord centerline detection}

We assessed the line centerline identification, through registering (I) the Mean Square Error (MSE) between the anticipated as well as manual spinal string center appearance, (ii) the restriction rate, characterized as the level of pivotal cuts in favour of which the anticipated centreline be incorporated into the physically sectioned spinal line. We produced the manual spinal rope centre appearance beside processing the focal point of collection of every hub cut of the instruction booklet spinal line divisions, normalize among an approximated non-uniform levelheaded Bezier spline, as portrayed in [10]. We looked at our spinal string discovery strategy (Figure 3, step 1-2) to an as of late distributed examination [11] that presented a worldwide bend streamlining calculation however utilized a prepared Support-Vector-Machine/-(SVM) calculation in the direction of create the spinal rope temperature map (rather than the CNN1 at Step 1). We allude to this as "SVM + OptiC" in the rest of this labour. A non-parametric examination [12] be connected to survey possible execution contrasts connecting these 2 methodologies.

\subsubsection{Spinal cord segmentation}

We evaluated the spinal rope division execution, by ascertaining (I) the Dice resemblance Coefficient moreover (ii) the comparative number contrast in fragmented amount (hilter kilter metric) connecting the programmed along with the guidebook division covers. We contrasted the spinal line division strategy with a formerly distributed unsupervised technique, PropSeg, which depends on multi-goals engendering of cylindrical deformable models. Kruskal-Wallis tests surveyed completing contrasts between the two techniques[20-22].

\subsubsection{MS lesion segmentation}

We evaluated the intra modularly M-S sore division execution (for illustration give way of C-NN/2-injury, observe Figure 3, stage/3), through computing (I) the Dice, (ii) the relation amount distinction, (iii) the voxel-intelligent affectability, also (iv) the voxel-sensible exactness among the programmed along with the labour-intensive division covers of the $\mathrm{M} * \mathrm{~S}$ associate. Voxel-wise dimensions measured a voxel as correctly separated beside the computation (in support of instance authentic constructive) on the off opportunity with the aim of it was name as "injury" through the raters[26].

We additionally figured the injury insightful affectability and the sore astute accuracy, where singular injuries (for example 3D associated articles) be insolvent down as elements (for example rather than each voxel independently, with respect to the voxel-wise measurements). We considered a competitor injury as accurately distinguished (for exemplar genuine positive) when the programmed division associated voxels covered with over $25 \%$ of the manual division voxels, else it was considered as mistakenly distinguished (for example false positive). On the off chance that an affirmed injury (for instance actually marked) had an inadequate cover $(<25 \%)$ with the programmed division voxels, at that point we characterized it as notidentified (for example copied unhelpful).The particularity of the programmed injury finder be processed taking place information commencing sound joystick as well as M-S patients who do not contain some intramodullary sore recognized, as well as call volume-shrewd explicitness in the staying of this broadsheet. We thought about a capacity as inaccurately recognized (for pattern false positive) if somewhere around one injury was naturally recognized. We expected solid control information to be without sore.

\subsubsection{Inter-rater variability of the MS lesion segmentation}

We evaluated the between quite fluctuation of injury division amongst every one taking an interest ratters $(n=7)$, on a randomized compartment of patients $(n=10)$. In support of every one of these patients, II sweeps be accessible, which enables the rates near segment the two production in equivalent by consolidating their data. In favour of this reason, we determined the Dice coefficient connecting apiece rater's division moreover an agreement perusing cover, delivered utilizing "dominant part casting a ballot" over all the raters' marks. 


\section{RESULTS}

Table 1 demonstrates the normal cover flanked by every couple of freely strained labour-intensive divisions. This sets up a standard in support of variety in guide divisions towards which the variety stuck between the guide in addition to programmed divisions (Table 2) canister be looked at. It is obvious commencing the deprived communication of the harmed rope divisions of discharge along with particularly pallid issue with the aim of blue-collar division can't survive indulgence as the ground truth, all things considered in a few works. Table 2 demonstrates the all out normal cover of our division grades among the guide dissection. On behalf of splitting up of the tissue locales of control spinal ropes, the execution of our calculation is all things considered proportionate to that of the master manual divisions. Correspondingly, for all separation of the harmed lines - the entire lines, the spinal line barring drain, and the white matte our programmed divisions are by and large in any event in the same class as the master manual divisions[23-24]. The outrageous absence of consistency between master divisions of harmed white issue makes it outlandish for our calculation to have a high cover with all specialists, yet thinking about the cover between master divisions, the relative execution is solid.
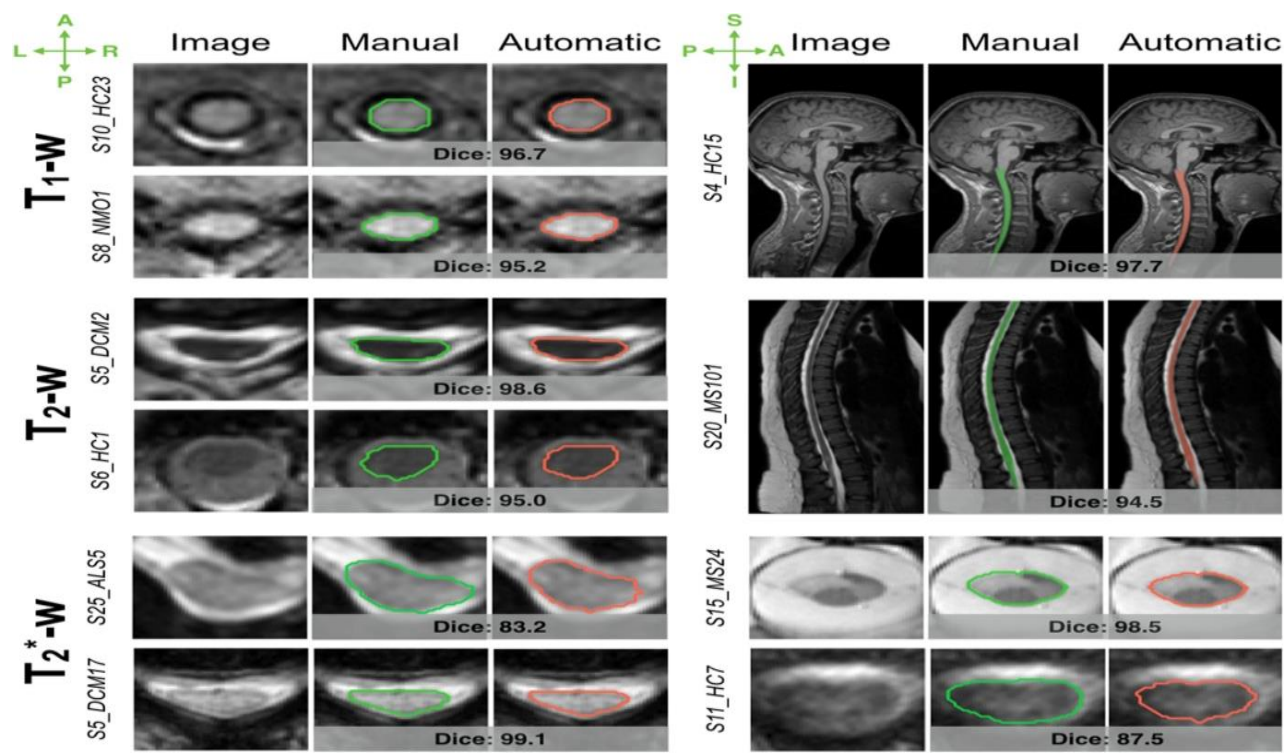

Figure 4. Disease finding using CNN

Table 1. Demonstrates the Normal Cover Flanked by Every Couple of Freely Strained Labour-Intensive Divisions

\begin{tabular}{|c|c|c|c|c|c|c|c|c|c|}
\hline \multicolumn{5}{|c|}{ A. Centreline Detection } & \multicolumn{5}{|c|}{ B. Spinal Cord Segmentation } \\
\hline & \multirow{2}{*}{\multicolumn{2}{|c|}{$\begin{array}{c}\text { Mean square error- } \\
{[\mathrm{mm}]} \\
\text { Best value: } 0\end{array}$}} & \multirow{2}{*}{\multicolumn{2}{|c|}{ Localization-rate $/[0,100] \%$}} & & \multirow{2}{*}{\multicolumn{2}{|c|}{$\begin{array}{c}\text { Dice coeffient }[0, \\
100] \% \\
\text { Best value:/-100 }\end{array}$}} & \multirow{2}{*}{\multicolumn{2}{|c|}{$\begin{array}{c}\text { Relation volume } \\
\text { dissimilarity \% } \\
\text { Best worth: } 0\end{array}$}} \\
\hline & & & & & & & & & \\
\hline & $\begin{array}{c}\mathrm{SVM}+\mathrm{Opt} \\
\mathrm{iC}\end{array}$ & $\begin{array}{c}\mathrm{CNN} 1+\mathrm{Opt} \\
\mathrm{iC}\end{array}$ & $\begin{array}{c}\mathrm{SVM}+\text { Opti } \\
\mathrm{C}\end{array}$ & $\begin{array}{l}\mathrm{CNN} 1+\mathrm{Op} \\
\text { ti C }\end{array}$ & & $\begin{array}{l}\text { Prop } \\
\text { Seg. }\end{array}$ & $\begin{array}{c}\text { C/N/N2- } \\
\text { SC }\end{array}$ & $\begin{array}{c}\text { PropSe } \\
\mathrm{g}\end{array}$ & $\begin{array}{c}\mathrm{CNN} 2- \\
\mathrm{SC}\end{array}$ \\
\hline $\begin{array}{l}\text { T1-w } \\
\text { data }\end{array}$ & 11.1-(11.8) & $0.9 *(0.5)$ & $33.3 /(48.9)$ & $100-(0)$ & $\begin{array}{l}\text { T1-w } \\
\text { data }\end{array}$ & $\begin{array}{l}92.0- \\
(13.5)\end{array}$ & $\begin{array}{l}95.9 / \\
(1.5)\end{array}$ & $\begin{array}{l}-4.4 / \\
(11.1)\end{array}$ & $\begin{array}{l}-0.3- \\
(5.7)\end{array}$ \\
\hline $\begin{array}{l}\text { T2-w } \\
\text { data }\end{array}$ & $9.1 /(12.8)$ & $1.0 /(0.9)$ & $100 /(33.3)$ & $99.7-(4.2)$ & $\begin{array}{l}\text { T2-w } \\
\text { data }\end{array}$ & $\begin{array}{l}83.2 / \\
(18.6)\end{array}$ & $\begin{array}{l}92.4- \\
(5.1)\end{array}$ & $\begin{array}{c}7.0 /(26 \\
.8)\end{array}$ & $\begin{array}{l}-0.2- \\
(6.5)\end{array}$ \\
\hline $\begin{array}{c}\mathrm{T} 2 * \text {-w } \\
\text { data }\end{array}$ & $0.9 /(0.3)$ & $1.0-(0.6)$ & $100 *(0)$ & $100-(0)$ & $\begin{array}{c}\mathrm{T} 2 * \text {-w } \\
\text { data }\end{array}$ & $\begin{array}{c}94.1 /(1 \\
5.7)\end{array}$ & $\begin{array}{l}95.5- \\
(2.8)\end{array}$ & $\begin{array}{c}4.3 /(32 \\
.8)\end{array}$ & $\begin{array}{l}-3.5 / \\
(9.8)\end{array}$ \\
\hline
\end{tabular}

Table 2. Demonstrates the All Out Normal Cover of Our Division Grades Among the Guide Dissection

\begin{tabular}{|c|c|c|c|c|c|c|c|}
\hline & $\begin{array}{c}\text { Dice/coff }[0, \\
100] \%\end{array}$ & $\begin{array}{c}\text { Relative- } \\
\text { volume } \\
\text { difference/ \% }\end{array}$ & $\begin{array}{c}\text { Lesion-wise } \\
\text { sensitivity*[0, } \\
100] \%\end{array}$ & $\begin{array}{c}\text { Lesion-wise } \\
\text { precision/-[0, } \\
100] \%\end{array}$ & $\begin{array}{c}\text { Voxel-wise } \\
\text { sensitivity/*[0, } \\
100] \%\end{array}$ & $\begin{array}{c}\text { Voxel-wise } \\
\text { precision-* }[0, \\
100] \%\end{array}$ & $\begin{array}{l}\text { Voxel-wise } \\
\text { specificity- } \\
/[0,100] \% \\
\end{array}$ \\
\hline & $\begin{array}{c}\text { Best-value:- } \\
100\end{array}$ & Best value:-0 & Best*value:100 & $\begin{array}{c}\text { Top value:- } \\
100\end{array}$ & $\begin{array}{c}\text { Best- } \\
\text { value*100 }\end{array}$ & $\begin{array}{c}\text { Best- } \\
\text { value/100 }\end{array}$ & $\begin{array}{c}\text { Greatest } \\
\text { value:-100 }\end{array}$ \\
\hline $\mathrm{T}_{2}$-W- data & $57.60-(22.4)$ & $-17.3 /(61.3)$ & $90.0 *_{-}(33.3)$ & $66.70-(58.3)$ & 51.4-(39.4) & $68.3 /(39.6)$ & 80.61 \\
\hline $\mathrm{T}_{2}{ }^{*}-\mathrm{w}-$ data & $60.4 /(25.0)$ & $-4.5 /(74.9)$ & $75.0-(47.2)$ & $100-(38.4)$ & $59.0 *(38.6)$ & $47.4-(59.2)$ & 81.52 \\
\hline
\end{tabular}




\section{CONCLUSION}

We comprise anticipated another multistep, CEM-supported way in the direction of deal with spinal string along with pallid issue division commencing in vivo M-R pictures along with we include approved with the intention of its execution is comparable to to facilitate of master manual division. We include exhibited with the aim of our calculation, in contrast to past methodologies, stays solid for spinal rope dissection within the sight of moderate and serious rope damage, not simply incredibly mellow damage. What's additional, we enclose exhibited to facilitate our calculation is as solid as the normal individual master in support of release moreover white issue division on behalf of harmed rat spinal strings. Prospect occupation incorporates fuse of a progressively point through indicate bodily representation on behalf of the spinal tissues, just as association of physical moreover automatic M/R-I division grades to spinal histology marks. We anticipate facilitating our calculation determination give way nearer concurrence among histology than a normal physical master division do. Prospect occupation additionally incorporates adjustment of the calculation to M-R pictures of creature spinal strings, which contain inferior goals than $M * R$ pictures of rat lines.

\section{REFERENCES}

[1] Scheff SW, Rabchevsky AG, Fugaccia I, Main JA, Lumpp JE. "Experimental Modeling of Spinal Cord Injury: Characterization of A Force-Defined Injury Device". J Neurotrauma 2003; 20: 179-193.

[2] Ma M, Basso DM, Walters P, Stokes BT, Jakeman LB. "Behavioral and Histological Outcomes Following Graded Spinal Cord Contusion Injury in the C57BL/6 Mouse". Exp Neurol 2001; 169: 239-254.

[3] Basso DM, Beattie MS, Bresnahan JC. "Descending Systems Contributing to Locomotor Recovery after Mild or Moderate Spinal Cord Injury in Rats: Experimental Evidence and A Review of Literature. Restor Neurol Neurosci 2002; 20: 189-218.

[4] Basso DM. "Neuroanatomical Substrates of Functional Recovery after Experimental Spinal Cord Injury: Implications of basic Science Research for Human Spinal Cord Injury". Phys Ther 2000; 80: 808-817.

[5] McEwen ML, Springer JE. "Quantification of Locomotor Recovery Following Spinal Cord Contusion in Adult Rats". J Neurotrauma 2006; 23: 1632-1653.

[6] Magnuson DS, Lovett R, Coffee C, Gray R, Han Y, Zhang YP, Burke DA. "Functional Consequences Of Lumbar Spinal Cord Contusion Injuries in the Adult Rat". J Neurotrauma 2005; 22: 529-543.

[7] Basso DM, Beattie MS, Bresnahan JC. "Graded Histological and Locomotor Outcomes after Spinal Cord Contusion using Thenyuweight-Drop Device Versus Transection”. Exp Neurol 1996; 139: 244-256.

[8] Kim JH, Loy DN, Liang HF, Trinkaus K, Schmidt RE, Song SK. "Noninvasive Diffusion Tensor Imaging of Evolving White Matter Pathology in A Mouse Model of Acute Spinal Cord Injury”. Magn Reson Med 2007; 58: 253-260.

[9] Loy DN, Kim JH, Xie M, Schmidt RE, Trinkaus K, Song SK. "Diffusion Tensor Imaging Predicts Hyperacute Spinal Cord Injury Severity". J Neurotrauma 2007; 24: 979-990.

[10] Cohen-Adad J, Benali H, Hoge RD, Rossignol S. "In Vivo DTI of the Healthy and Injured Cat Spinal Cord at High Spatial and Angular Resolution". Neuroimage 2008; 40: 685-697.

[11] Schwartz ED, Duda J, Shumsky JS, Cooper ET, Gee J. "Spinal Cord Diffusion Tensor Imaging and Fiber Tracking can Identify White Matter Tract Disruption and Glial Scar Orientation Following Lateral Funiculotomy". J Neurotrauma 2005; 22: 1388-1398.

[12] Kass M, Witkin A, Terzopoulos D. "Snakes: Active Contour Models". Int J Computer Vision 1987;1:259-268.

[13] Osher S, Sethian JA. "Fronts Propagating with Curvature Dependent Speed: Algorithms based on Hamilton-Jacobi Formulations". J Comput Phys 1988; 79: 12-49.

[14] Deng X, Ramu J, Narayana PA. "Spinal Cord Atrophy in Injured Rodents: High-Resolution MRI”. Magn Reson Med 2007; 57: 620-624.

[15] Ellingson BM, Ulmer JL, Schmit BD. "Gray and White Matter Delineation in the Human Spinal Cord using Diffusion Tensor Imaging and Fuzzy Logic". Acad Radiol 2007; 14: 847-858.

[16] Ellingson BM, Ulmer JL, Schmit BD. "Morphology and Morphometry of Human Chronic Spinal Cord Injury using Diffusion Tensor Imaging and Fuzzy Logic”. Ann Biomed Eng 2008; 36: 224-236.

[17] Younis AA, Ramirez N, Pattany PM, Burns RJ, Sharawy MI. "Automated Segmentation of Spinal Diffusion Tensor MR Imaging”. In: IEEE SoutheastCon, Fort Lauderdale, Florida, 2005. Pp. 187-192.

[18] Budde MD, Kim JH, Liang HF, Russell JH, Cross AH, Song SK. "Axonal Injury Detected by in Vivo Diffusion Tensor Imaging Correlates with Neurological Disability in A Mouse Model of Multiple Sclerosis". NMRBiomed 2007; 21: 589-597.

[19] DeBoy CA, Zhang J, Dike S, Shats I, Jones M, Reich DS, Mori S, Nguyen T, Rothstein B, Miller RH, Griffin JT, Kerr DA, Calabresi PA. "High Resolution Diffusion Tensor Imaging of Axonal Damage in Focal Inflammatory and Demyelinating Lesions in Rat Spinal Cord". Brain 2007; 130: 2199-2210.

[20] Gullapalli J, Krejza J, Schwartz ED. "In Vivo DTI Evaluation of White Matter Tracts in Rat Spinal Cord". J Magn Reson Imaging 2006; 24: 231-234.

[21] Celeux G, Govaert G. "A Classification EM Algorithm for Clustering and Two Stochastic Versions". Comput Stat Data Anal 1992; 14: 315-332. 
[22] Dempster A, Laird N, Rubin D. "Maximum Likelihood from Incomplete Data Via the EM Algorithm". J R Stat Soc Ser B 1977; 39: 1-38.

[23] Jakeman LB, Guan Z, Wei P, Ponnappan R, Dzwonczyk R, Popovich PG, Stokes B. Traumatic Spinal Cord Injury Produced by Controlled Contusion in Mouse". J Neurotrauma 2000; 17: 299-319.

[24] Stejskal EO, Tanner JE. "Spin Diffusionmeasurements: Spin Echoes in the Presence of A Time-Dependent Field Gradient". J Chem Phys 1965; 42: 288-292.

[25] Koay CG, Chang LC, Carew JD, Pierpaoli C, Basser PJ. "Aunifying Theoretical and Algorithmic Framework for Least Squares Methods of Estimation in Diffusion Tensor Imaging”. J Magn Reson 2006; 182: 115-125.

[26] Sk.Hasane Ahammad,V.Rajesh, "Image Processing based Segmentation for Spinal Cord in MRI", Indian Journal of Public Health Research and Development 9(6), pp. 317-323. 